\title{
Changes in body composition in patients with malignant pleural mesothelioma and the relationship with activity levels and dietary intake
}

\author{
Emily Jeffery ${ }^{1,2,3 凶}$, Y. C. Gary Lee ${ }^{4,5,6}$, Robert U. Newton (iD ${ }^{1,2}$, Philippa Lyons-Wall ${ }^{2}$, Joanne McVeigh ${ }^{7,8}$, Deirdre B. Fitzgerald ${ }^{4}$, \\ Leon Straker (D) ${ }^{7}$ and Carolyn J. Peddle-Mclntyre ${ }^{1,2}$
}

(c) The Author(s) 2022

BACKGROUND: Skeletal muscle loss is common in advanced cancer and is associated with negative outcomes. In malignant pleural mesothelioma (MPM), no study has reported body composition changes or factors associated with these changes. This study aimed to describe changes in body composition over time and its relationship with activity levels, dietary intake and survival.

METHODS: The study was a secondary analysis of data collected from a longitudinal observational study of patients with MPM. Participants completed 3-month assessments for up to 18 months. Participants with two dual-energy x-ray absorptiometry (DXA) scans were included. Changes in appendicular skeletal muscle mass (ASM) and total fat mass were used to categorise participants into phenotypes. Activity levels were measured with an ActiGraph GT3X+ accelerometer and energy and protein intake was measured with a 3-day food record and 24-h recall.

RESULTS: Eighteen participants were included (89\% men, mean age $68.9 \pm 7.1$ years). Median time between DXA was 91 [IQR 84-118] days. Compared to participants with ASM maintenance $(n=9)$, fewer participants with ASM loss $(n=9)$ survived $\geq 12$ months from follow-up $(p=0.002)$. Participants with ASM loss increased sedentary time $(p=0.028)$ and decreased light activity $(p=0.028)$ and step count $(p=0.008)$. Activity levels did not change in participants with ASM maintenance $(p>0.05)$. Energy and protein intake did not change in either group $(p>0.05)$.

CONCLUSIONS: Muscle loss was associated with poorer survival and decreased activity levels. Interventions that improve physical activity or muscle mass could benefit patients with MPM.

European Journal of Clinical Nutrition (2022) 76:979-986; https://doi.org/10.1038/s41430-021-01062-6

\section{INTRODUCTION}

Malignant pleural mesothelioma (MPM) is an incurable cancer that results from asbestos exposure [1]. Patients with MPM have limited treatment options and short median survival of 12 months [1]. It has been hypothesised that cancer cachexia could contribute to the cause of death in MPM [2].

Cancer cachexia is a form of malnutrition characterised by the loss of skeletal muscle mass in the presence or absence of loss of fat mass, and is often accompanied by anorexia and systemic inflammation [3]. Cancer cachexia can lead to the development of low skeletal muscle mass, which is associated with a range of negative outcomes in advanced cancer populations including poorer quality of life [4], lower activity levels [5], increased treatment toxicity [6], and poorer survival [6]. Further, people with both low skeletal muscle mass and excess fat mass (i.e., sarcopenic obese) have had a greater risk of negative outcomes [7].
In our previous research, we reported that $50 \%$ of patients with MPM had low skeletal muscle mass close to the time of diagnosis, and of these participants, $11 \%$ were obese [5]. While anecdotally clinicians report that patients with MPM become emaciated over the disease course, and often die with a low BMI [2], there is a lack of evidence available on changes in body composition over time to inform the development of interventions to address these concerns.

Physical activity and dietary intake are modifiable factors that could be central to the development of cancer cachexia. Physical activity and dietary protein intake stimulate muscle protein synthesis [8] and in sufficient quantities could protect against the development of low skeletal muscle mass $[9,10]$. In addition, lower levels of physical activity and high dietary energy intake can create a positive energy balance resulting in weight gain that is largely an increase in fat mass [11]. At present, there is little research on the relationship between physical activity, dietary intake and changes in body composition in cancer populations.

\footnotetext{
${ }^{1}$ Exercise Medicine Research Institute, Edith Cowan University, Joondalup, WA, Australia. ${ }^{2}$ School of Medical and Health Sciences, Edith Cowan University, Joondalup, WA, Australia. ${ }^{3}$ School of Population Health, Curtin University, Perth, WA, Australia. ${ }^{4}$ Respiratory Department, Sir Charles Gairdner Hospital, Nedlands, WA, Australia. ${ }^{5}$ Institute for Respiratory Health, Nedlands, WA, Australia. ${ }^{6}$ Medical School, University of Western Australia, Crawley, WA, Australia. ${ }^{7}$ School of Allied Health, Curtin University, Perth, WA, Australia. ${ }^{8}$ Movement Physiology Laboratory, School of Physiology, University of Witwatersrand, Johannesburg, South Africa. ${ }^{\circledR}$ email: emily.jeffery@curtin.edu.au
} 
Understanding these relationships could lead to more targeted interventions to prevent and treat cancer cachexia. Therefore, this study in patients with MPM aimed to describe the changes in body composition over time and the relationship between body composition changes and activity levels and dietary intake. A secondary aim of the study was to explore the association between body composition changes and participant characteristics, such as survival.

\section{METHODS \\ Study design and participants}

The study was a secondary analysis of data collected from a longitudinal observational study that aimed to describe the functional and nutritional status of patients with MPM. Participants were recruited from a pleural disease clinic in Perth, Western Australia. Patients were eligible if they had cytological or histological confirmation of MPM. Patients were excluded if they were aged $<18$ years, pregnant or lactating, unable to read and understand English, unable to comply with the protocol or participating in an intervention study likely to influence body composition. Participant consent and physician approval were required for participation in the study. Participants completed assessments of body composition, activity levels and dietary intake during routine hospital visits, approximately every 3 months and were followed until death or for a maximum of 18 months. Participants that did not complete body composition scans at two consecutive assessments were excluded from this analysis. The study was approved by the Sir Charles Gairdner Group and Edith Cowan University Human Research Ethics Committees (ID: 2014-124 and 13255).

\section{Measurements}

Demographic and medical variables. Demographic and medical data were obtained from participant medical records. Disease progression at followup was determined by clinician examination of the computed tomography scan completed closest to the second body composition scan. The Eastern Cooperative Oncology Group (ECOG) performance status was recorded on the date of assessment [12].

Anthropometric measures. Weight and height, measured with participants dressed in light clothing with shoes removed, were used to calculate the BMI. Participants were classified as underweight, normal weight, overweight or obese based on World Health Organisation BMI criteria [13].

Body composition. Body composition was assessed using whole-body dual-energy $x$-ray absorptiometry (DXA) scans (Hologic Horizon A, Hologic Inc., Marlborough, MA, USA). DXA is considered a precise measure of body composition, with coefficients of variation reported to be $<0.5 \%$ for lean mass, and $<1.0 \%$ for fat mass [14]. Device calibration was completed daily using the Hologic Spine Phantom [15]. Participants wore light clothing with shoes removed, and were asked to remove all metal objects (i.e., glasses, jewellery). Participants were positioned in a supine position in the centre of the table, their arms by their side and palms facing down, with legs were shoulder-width apart and internally rotated [16]. Participants who were unable to lie flat were given a pillow to support their head [15]. Following the scan, measured weight was compared to total mass to check for any discrepancies. DXA scans were analysed using the in-built scan analysis software (version 13.5.2). To analyse appendicular skeletal muscle and fat mass, a single researcher (EJ) manually corrected the separation of body regions, so that the arms were separated at the acromio-humeral joints and the legs were separated at the pelvic-femoral joints [17]. Low skeletal muscle mass was defined as BMl-adjusted appendicular skeletal muscle mass of $0.86 \mathrm{~kg} / \mathrm{kg} / \mathrm{m}^{2}$ for men and $0.59 \mathrm{~kg} / \mathrm{kg} / \mathrm{m}^{2}$ for women [18]. The cut-points for appendicular skeletal muscle mass were derived from Hologic DXA devices and set as two standard deviations below the mean of a reference sample of young Australian adults [18]. Participants with low skeletal muscle mass were categorised as having low muscularity [19]. Change in body composition variables was calculated as the per cent change between the second and first measurements.

To characterise changes in body composition over time, participants were categorised into body composition phenotypes according to changes in skeletal muscle mass and fat mass. The total lean mass measured with DXA includes both skeletal muscle and residual mass (i.e., organs); however, appendicular lean mass is predominantly skeletal muscle [20]. Therefore, to report on changes in skeletal muscle mass we used appendicular lean mass, known as appendicular skeletal muscle mass, which represents on average, $75 \%$ of whole-body skeletal muscle [20]. The four body composition phenotypes were: (1) loss of appendicular skeletal muscle mass and loss of total fat mass; (2) loss of appendicular skeletal muscle mass and maintenance or gain of total fat mass; (3) maintenance or gain of appendicular skeletal muscle mass and loss of total fat mass and (4) maintenance or gain of appendicular skeletal muscle mass and maintenance or gain of total fat mass. A loss was defined as a change of $\geq-1.45 \%$ for appendicular skeletal muscle mass and $\geq-2.15 \%$ for total fat mass between the first and second measurements; maintenance or gain was defined as a change of $<-1.45 \%$ for appendicular skeletal muscle mass and $<-2.15 \%$ for total fat mass between the first and second measurements. These cut-points represent the minimal detectable change values reported for total lean and fat mass in a study of Hologic DXA devices [14].

Activity levels. Sedentary behaviour and physical activity were devicederived following each body composition scan using the ActiGraph GT3X+ accelerometer (Actigraph, Pensacola, FL, USA). The ActiGraph GT3X+ accelerometer has been shown to have high intra- and inter-device reliability, with coefficients of variation $\leq 2.5 \%$ and an intraclass coefficient of 0.99 [21]. Participants were instructed to wear the accelerometer on their hip $24 \mathrm{~h}$ /day for 3 days, to only remove for bathing or swimming and to record any non-wear time in a logbook. Cut-points were applied to classify sedentary behaviour as $<100$ counts/minute (cpm), light activity as $100-1952 \mathrm{cpm}$ and moderate and vigorous physical activity (MVPA) as $>1952 \mathrm{cpm}[22,23]$. Variables were calculated per day and then averaged across all valid (at least $10 \mathrm{~h}$ of data) days. Additional accelerometer methodology for this study has been reported previously [5].

Dietary intake. Dietary intake was measured following each body composition scan using a 3-day estimated food record at the initial assessment and a $24-\mathrm{h}$ recall at subsequent assessments. These dietary assessment methods are reported to accurately measure energy and protein intake $[24,25]$. Dietary intake data were collected, verified and analysed by a dietitian with experience in dietary intake assessment. To assist participants with the completion of the food record, written and verbal instructions were provided explaining how to complete the food record and estimate portion sizes using household measures. Returned food records were visually inspected and incomplete details were clarified with participants. Participants completed the 24-h recall in a face-to-face interview with a dietitian. 24-h recalls were conducted per the multiple pass method [25]. The food records and 24-h recalls were analysed using Foodworks 8 software (Xyris Software Pty Ltd, Queensland, Australia). Intake variables were calculated per day, and for the food records, intake was averaged across all days. Energy and protein intake were expressed as $\mathrm{kJ}$ or $\mathrm{g}$ per $\mathrm{kg}$ of body weight per day.

\section{Statistical analyses}

Statistical analyses were conducted using the Statistical Package for the Social Sciences (v. 23, IBM Corporation, Somers, NY, USA). Data were expressed as mean (SD) or median [IQR] where the data were not normally distributed. To examine the relationship between changes in body composition and participant characteristics, activity levels and dietary intake, participants were condensed into two groups: (1) participants with a change of $\geq-1.45 \%$ in appendicular skeletal muscle mass, defined as the muscle loss group; and (2) participants with a change of $<-1.45 \%$ in appendicular skeletal muscle mass, defined as the muscle maintenance group. Fisher's exact test was used to test for differences in characteristics between participants with muscle loss and muscle maintenance where the data were categorical. As the data were not normally distributed, the Mann-Whitney test was used to test for differences in characteristics between participants with muscle loss and muscle maintenance where the data were continuous, and for differences in change in activity levels and dietary intake between muscle groups. The Wilcoxon signed-rank test was used to test for differences in body composition, activity levels and dietary intake between the first and second measurements.

\section{RESULTS}

\section{Participant characteristics}

Of the 36 patients recruited to the longitudinal observational study, $18(50 \%)$ were included in this study (Fig. 1). The median time between the first and second body composition scans was 91 [84-118] days. Participant characteristics are presented in Table 1. 


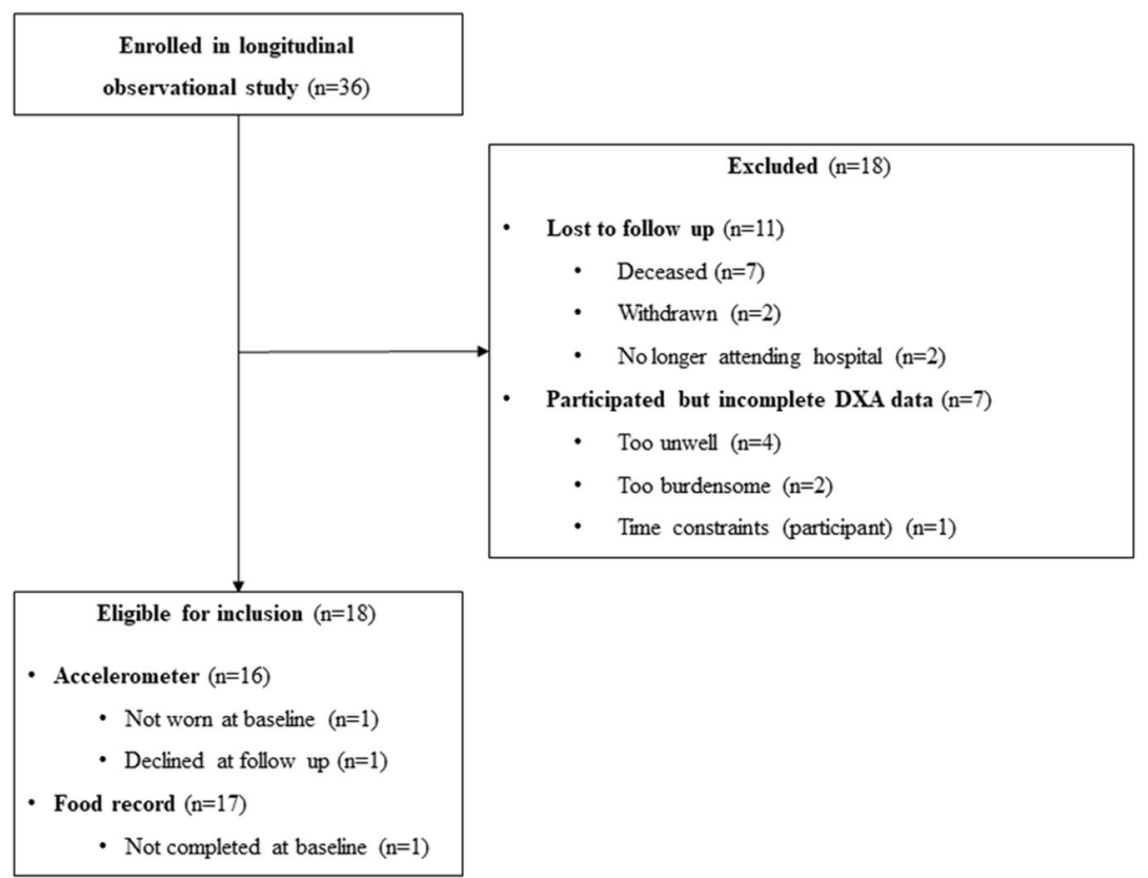

Fig. 1 Study flowchart. Participants included and excluded in the study.

Table 1. Participant characteristics, $n=18$.

\begin{tabular}{|c|c|c|c|c|}
\hline & All participants $(n=18)$ & Muscle loss $(n=9)$ & Muscle maintenance $(n=9)$ & $p$ value \\
\hline Age, years & $68.9 \pm 7.1$ & $65.0[61.0-74.5]$ & $69.0[63.0-75.0]$ & 0.605 \\
\hline Sex, men & $16(88.9 \%)$ & $8(88.9 \%)$ & $8(100 \%)$ & 0.471 \\
\hline$B M I, \mathrm{~kg} / \mathrm{m}^{2}$ & $25.2[23.9-28.7]$ & $26.1[23.9-29.8]$ & $24.6[23.8-27.1]$ & 0.258 \\
\hline \multicolumn{5}{|l|}{ BMI category } \\
\hline Underweight & $0(0.0 \%)$ & $0(0.0 \%)$ & $0(0.0 \%)$ & \multirow[t]{4}{*}{-} \\
\hline Normal weight range & $9(50.0 \%)$ & $3(33.3 \%)$ & $6(66.6 \%)$ & \\
\hline Overweight & $7(38.9 \%)$ & $4(44.4 \%)$ & $3(33.3 \%)$ & \\
\hline Obese & $2(11.1 \%)$ & $2(22.2 \%)$ & $0(0.0 \%)$ & \\
\hline Low muscularity, yes & $11(61.1 \%)$ & $6(66.6 \%)$ & $5(55.6 \%)$ & 1.000 \\
\hline Histological subtype, epithelioid & $15(83.3 \%)$ & $7(77.8 \%)$ & $8(88.9 \%)$ & 1.000 \\
\hline ECOG performance status at first scan ${ }^{\mathrm{a}}, 0-1$ & $18(100 \%)$ & $9(100 \%)$ & $9(100 \%)$ & - \\
\hline \multicolumn{5}{|l|}{ Time from diagnosis to first scan ${ }^{\mathrm{a}}$} \\
\hline$<3$ months & $10(55.6 \%)$ & $4(44.4 \%)$ & $6(66.6 \%)$ & \multirow[t]{3}{*}{-} \\
\hline 3-12 months & $5(27.8 \%)$ & $3(33.3 \%)$ & $2(22.2 \%)$ & \\
\hline$>12$ months & $3(16.7 \%)$ & $2(22.2 \%)$ & $1(11.1 \%)$ & \\
\hline Time from first to second scan, days & $91.0[84.0-118.0]$ & $85.0[81.0-94.0]$ & $98.0[87.0-140.0]$ & 0.113 \\
\hline Cancer treatment during follow-up, yes & $9(50.0 \%)$ & $4(44.0 \%)$ & $5(55.6 \%)$ & 1.000 \\
\hline \multicolumn{5}{|l|}{ Type of cancer treatment } \\
\hline Cisplatin and pemetrexed & $3(33.3 \%)$ & $1(25.0 \%)$ & $2(40.0 \%)$ & \multirow[t]{4}{*}{-} \\
\hline Carboplatin and pemetrexed & $3(33.3 \%)$ & $1(50.0 \%)$ & $2(40.0 \%)$ & \\
\hline Vinorelbine & $1(11.1 \%)$ & $1(25.0 \%)$ & $0(0.0 \%)$ & \\
\hline Clinical trial-cisplatin, pemetrexed, and durvalumab & $2(22.2 \%)$ & $1(25.0 \%)$ & $1(20.0 \%)$ & \\
\hline \multicolumn{5}{|l|}{ Disease progression at second scan ${ }^{\mathrm{a}}$} \\
\hline Progressed & $10(55.6 \%)$ & $5(55.6 \%)$ & $5(55.6 \%)$ & \multirow[t]{4}{*}{-} \\
\hline Stable & $4(22.2 \%)$ & $2(22.2 \%)$ & $2(22.2 \%)$ & \\
\hline Response to treatment & $2(11.1 \%)$ & $0(0.0 \%)$ & $2(22.2 \%)$ & \\
\hline Data not available & $2(11.1 \%)$ & $2(22.2 \%)$ & $0(0.0 \%)$ & \\
\hline \multicolumn{5}{|l|}{ Time from second scan ${ }^{\mathrm{a}}$ to death } \\
\hline$<12$ months & $7(38.9 \%)$ & $7(77.8 \%)$ & $0(0.0 \%)$ & \multirow[t]{2}{*}{0.002} \\
\hline$\geq 12$ months & $11(61.1 \%)$ & $2(22.2 \%)$ & $9(100.0 \%)$ & \\
\hline
\end{tabular}

${ }^{\mathrm{a}}$ First or second body composition scan. 


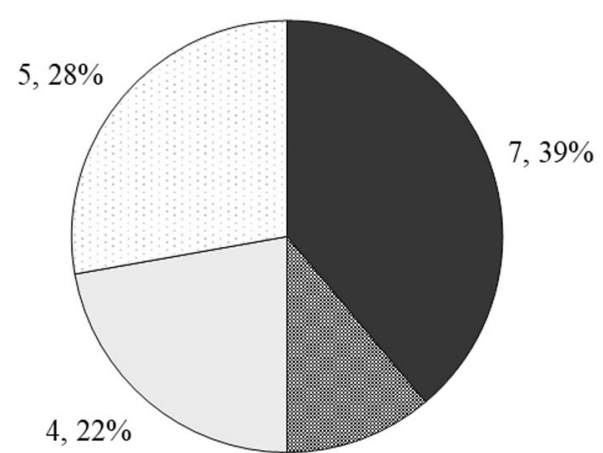

$2,11 \%$
- Loss of appendicular skeletal muscle and fat mass

임 Loss of appendicular skeletal muscle and maintenance or gain of fat mass

$\square$ Maintenance or gain of appendicular skeletal muscle and loss of fat mass

$\square$ Maintenance or gain of appendicular skeletal muscle and fat mass

Fig. 2 Proportion of participants within each body composition phenotype. (1) Loss of appendicular skeletal muscle mass and loss of total fat mass; (2) loss of appendicular skeletal muscle mass and maintenance or gain of total fat mass; (3) maintenance or gain of appendicular skeletal muscle mass and loss of total fat mass; and (4) maintenance or gain of appendicular skeletal muscle mass and maintenance or gain of total fat mass.

The majority of participants were men (89\%) and the mean age of participants was 68.9 (7.1) years. Nine participants (50\%) received cancer treatment during the follow-up period. Nine participants (50\%) met the criteria for muscle loss and nine participants (50\%) met the criteria for muscle maintenance. Seven participants (78\%) with muscle loss survived less than 12 months from the second body composition scan, while none of the participants (0\%) with muscle maintenance survived less than 12 months from the second body composition scan $(p=0.002)$. For some outcomes presented (e.g., cancer treatment status) the sample size was too small to complete statistical analysis. No other differences in participant characteristics were observed between muscle change groups $(p>0.05)$ (Table 1).

\section{Changes in muscularity status}

Ten participants (56\%) had low muscularity at the first and second body composition scans. One participant (14\%) with normal muscularity at the first measurement had low muscularity at the second scan. One participant (9\%) who had low muscularity at the first measurement had normal muscularity at the second scan. Of the participants with low muscularity, two (18\%) were obese.

\section{Changes in body composition}

When participants were condensed into the four body composition phenotypes, seven participants (39\%) had a loss of appendicular skeletal muscle and fat mass, two participants (11\%) had a loss of appendicular skeletal muscle and maintained fat mass, four participants (22\%) maintained appendicular skeletal muscle and lost fat mass and, five participants (28\%) maintained appendicular skeletal muscle and fat mass (Fig. 2).

There were no significant changes in total, lean or fat mass during the follow-up period in the whole group (Table 2). Participants with muscle loss $(n=9)$ experienced a significant decrease in total mass $(p=0.008)$, trunk lean mass $(p=0.011)$, appendicular skeletal muscle mass $(p=0.008)$ and trunk fat mass $(p=0.021)$ but not appendicular fat mass $(p=0.859)$. Participants with muscle maintenance $(n=9)$ experienced a significant increase in appendicular skeletal muscle mass $(p=0.038)$ but not total mass $(p=0.31)$ trunk lean mass $(p=0.594)$, trunk fat mass $(p=0.767)$ or appendicular fat mass $(p=0.594)$.

\section{Change in activity levels according to muscle change group}

There were no significant changes in activity levels during the follow-up period in the whole group (Table 3). Participants with muscle loss had a significant decrease in median step count ( $p=$ $0.008)$, an increase in the proportion of waking hours spent as sedentary $(p=0.028)$ and a decrease in the proportion of waking hours spent in light activity $(p=0.028)$ (Table 3$)$. There was no significant change in the proportion of waking hours spent in MVPA $(p=0.260)$. Participants with muscle maintenance maintained step count $(p=0.176)$, the proportion of waking hours spent as sedentary $(p=0.499)$, in light activity $(p=0.499)$ and in MVPA ( $p=0.176)$ (Table 3 ).

There was a significant difference between participants with muscle loss and muscle maintenance for change in step count $(-1020$ [ -4667 to 56$]$ vs. 1234 [ -204 to 2221 ] steps/day; $p=0.008$; Fig. 3) and for the proportion of waking hours spent in light activity $(-4.8$ [ -9.2 to 0.2$]$ vs. $-0.7[-2.0$ to 7.5$] ; p=0.023$; Fig. 3$)$ but not for the proportion of waking hours spent as sedentary (4.9 [ -2.3 to 11.1$]$ vs. 0.5 [ -8.6 to 2.2$] ; p=0.142 ;$ Fig. 3 ).

Change in dietary intake according to muscle change group There were no significant within-group changes in energy and protein intake during the follow-up period in the whole group (Table 3). Participants with muscle loss and those with muscle maintenance had no significant change in energy and protein intake (Table 3).

There was a significant difference between participants with muscle loss and muscle maintenance for change in protein intake $(-0.29$ [ -0.55 to 0.02$]$ vs. 0.60 [ -0.03 to 0.81$] \mathrm{g} / \mathrm{kg} / \mathrm{day} ; p=0.011$; Fig. 3) but not for change in energy intake $(-5.8[-46.1$ to -0.03$]$ vs. $9.4[-22.5$ to 103.6$] \mathrm{kJ} / \mathrm{kg} /$ day; $p=0.236$; Fig. 3).

\section{DISCUSSION}

Our study is the first to prospectively assess changes in body composition in relation to activity levels and dietary intake in patients with MPM. We identified multiple patterns of body composition change among our participants. Notably, participants with muscle loss and muscle maintenance had distinct survival, physical activity and dietary intake characteristics.

Our participants could be categorised across all four body composition phenotypes. The most common phenotype, which included $39 \%$ of participants, was the loss of appendicular skeletal muscle mass and fat mass, which is consistent with the cachexia phenotype [3]. When we condensed these body composition phenotypes to two groups: (1) muscle loss and (2) muscle maintenance; $50 \%$ of participants had muscle loss and $50 \%$ had muscle maintenance. This result is particularly notable as the low mean BMI reported in a post-mortem study indicates patients with MPM become emaciated over the disease course [2]. While muscle loss was common, our results suggest that a proportion of participants with MPM can maintain muscle, at least for a proportion of the disease course.

There were significant differences in survival between participants with muscle loss and muscle maintenance. Only a small 
proportion (22\%) of participants with muscle loss survived at least 12 months from the second body composition scan, while all (100\%) participants with muscle maintenance survived at least 12 months from the second body composition scan. Therefore, muscle loss could be indicative of shorter survival in patients with MPM. Similar findings have been reported in a large retrospective study of patients with advanced cancer $(n=368)$ [26] where the authors stated that muscle loss became more common as death approached [26]. Tumour burden is thought to mediate the metabolic changes that cause loss of muscle and fat mass [27], highlighting the importance of efficacious cancer treatments for the management of cachexia [3]. There are currently limited treatment options for those with MPM and only $40 \%$ of patients respond to first-line chemotherapy treatment [28]. Therefore, addressing lifestyle factors that contribute to cancer cachexia could offer benefits to patients with MPM.

Participants with muscle loss had a significant decline in activity levels over the follow-up period of 3 months, while participants with muscle maintenance maintained their activity levels. As physical activity is required for muscle protein synthesis [8], a decrease in physical activity may have contributed to muscle loss among our participants. In addition, as the majority of participants (70\%) with muscle loss had low muscularity at followup, participants may not have had the strength and endurance to participate in their usual physical activity. The lack of physical activity could result in an even greater reduction in muscle loss. Therefore, regardless of the causal pathway between muscle loss and activity levels, resistance exercise training may offer benefit to patients with MPM as it can improve skeletal muscle mass, strength and physical function [29].

There were no statistically significant changes in dietary intake over the follow-up period for participants with muscle loss and muscle maintenance; however, we made clinically meaningful observations. Participants with muscle loss had a median energy and protein intake that was within the recommended energy and protein intake range of $105-126 \mathrm{~kJ} / \mathrm{kg}$ and $1.0-1.5 \mathrm{~g} /$ day, respectively [30], while median energy and protein intake among participants with muscle maintenance exceeded these recommendations. A larger study $(n=52)$ in patients with incurable non-small cell lung cancer (NSCLC) reported that higher energy and protein intakes (149 kJ/kg and $1.4 \mathrm{~g} / \mathrm{kg}$, respectively) were associated with maintenance of skeletal muscle mass during chemotherapy [31]. Approximately $40-50 \%$ of patients with NSCLC are reported to have an elevated resting energy expenditure [32, 33], which could lead to muscle and fat loss unless dietary intake is increased proportionally. As muscle loss developed in our participants meeting dietary intake recommendations, it is possible that elevated resting energy expenditure exists to some extent in patients with MPM. Intakes of energy and protein that exceed recommendations may be needed to preserve skeletal muscle mass in patients with MPM.

This study has several potential limitations worthy of consideration. There are several factors known to affect muscle and fat metabolism, including disease progression, inflammation, cancer treatment and older age [34]. As participants in this study may be at different stages of the disease, combining all participants together could have introduced bias to the findings. While these characteristics were compared between participants with and without muscle loss, the sample size was too small to allow further evaluation concerning changes in body composition. Energy and protein intake at baseline and follow-up were measured using different dietary assessment methods, which could have affected the repeatability of the measurement. Participant feedback indicated that a 3-day food record was burdensome; therefore, we used 24-h recalls at follow-up assessments. Compared with a 24-h recall, a 3-day food record could be more representative of usual dietary intake as the measurement is carried out over a greater number of days; 
Table 3. Participant activity levels and dietary intake, $n=17$.

\begin{tabular}{|c|c|c|c|c|c|c|c|c|c|}
\hline & \multicolumn{3}{|c|}{ All participants $(n=17)$} & \multicolumn{3}{|c|}{ Muscle loss $(n=9)$} & \multicolumn{3}{|c|}{ Muscle maintenance $(n=8)$} \\
\hline & First scan & Second scan & $p$ value & First scan & Second scan & $p$ value & First scan & Second scan & $p$ value \\
\hline \multicolumn{10}{|c|}{ Activity behaviours } \\
\hline $\begin{array}{l}\text { Sedentary } \\
\text { behaviour, \% }\end{array}$ & $\begin{array}{l}70.3 \\
(61.7-73.1)\end{array}$ & $\begin{array}{l}73.1 \\
(64.0-76.0)\end{array}$ & 0.196 & $\begin{array}{l}67.0 \\
(58.1-72.7)\end{array}$ & $\begin{array}{l}73.7 \\
(66.8-84.2)\end{array}$ & $0.028^{*}$ & $\begin{array}{l}72.6 \\
(62.8-73.2)\end{array}$ & $\begin{array}{l}73.1 \\
(63.4-75.7)\end{array}$ & 0.499 \\
\hline MVPA, \% & $0.8(0.5-3.1)$ & $0.9(0.7-1.7)$ & 0.836 & $1.0(0.4-5.3)$ & $0.7(0.3-1.5)$ & 0.260 & $0.6(0.5-1.4)$ & $1.7(0.8-1.7)$ & 0.398 \\
\hline \multicolumn{10}{|l|}{ Dietary intake } \\
\hline $\begin{array}{l}\text { Energy } \\
\text { intake, } \mathrm{kJ} / \mathrm{kg}\end{array}$ & $\begin{array}{l}129.6 \\
(90.1-143.8)\end{array}$ & $\begin{array}{l}121.8 \\
(102.3-147.2)\end{array}$ & 0.981 & $\begin{array}{l}122.0 \\
(83.2-138.6)\end{array}$ & $\begin{array}{l}116.2 \\
(81.7-127.4)\end{array}$ & 0.314 & $\begin{array}{l}136.8 \\
(103.1-151.1)\end{array}$ & $\begin{array}{l}142.0 \\
(110.2-245.5)\end{array}$ & 0.263 \\
\hline $\begin{array}{l}\text { Protein } \\
\text { intake, } \mathrm{g} / \mathrm{kg}\end{array}$ & $1.5(0.9-2.0)$ & $1.4(1.1-1.8)$ & 0.492 & $1.0(0.9-1.9)$ & $1.2(0.7-1.4)$ & 0.260 & $1.5(1.0-2.0)$ & $1.7(1.4-2.7)$ & 0.069 \\
\hline
\end{tabular}

Data are presented as median (interquartile range). MVPA moderate and vigorous physical activity. ${ }^{*} p<0.05$.

a) Steps

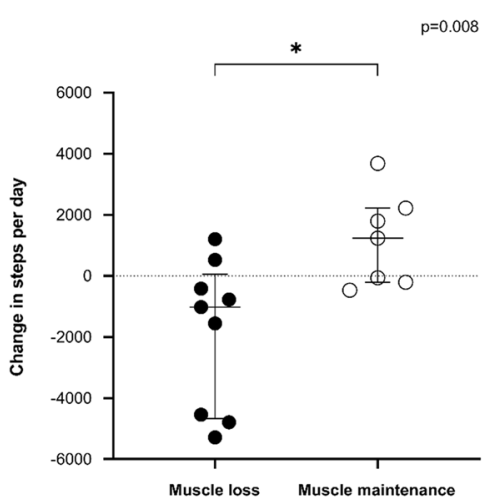

d) Energy intake

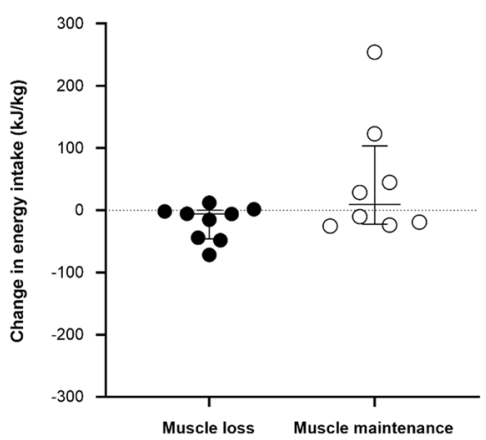

b) Sedentary behaviour

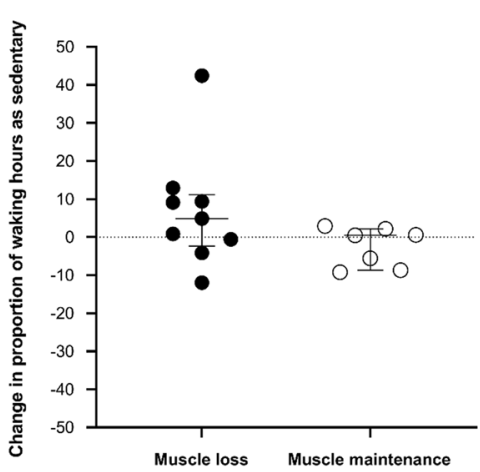

c) Light activity

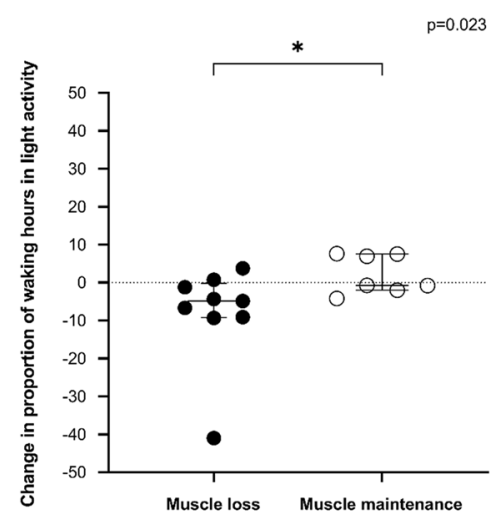

\section{e) Protein intake}

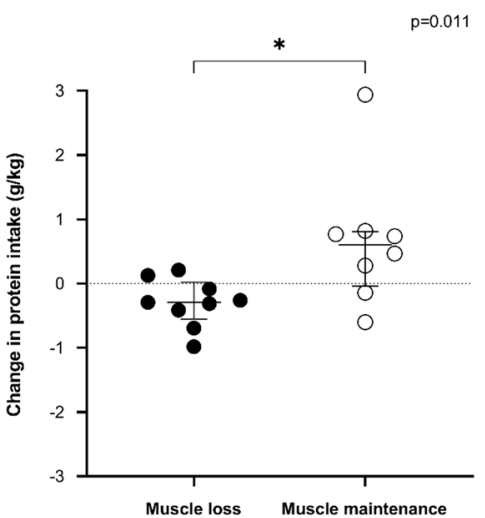

Fig. 3 Change in physical activity and dietary intake according to muscle change group. a Step count; $\mathbf{b}$ proportion of waking hours as sedentary; c proportion of waking hours in light activity; d energy intake $(\mathrm{kJ} / \mathrm{kg})$; and e protein intake $(\mathrm{g} / \mathrm{kg}) .{ }^{*} p<0.05$.

however, a 24-h food recall is not less accurate than a food record [25]. Considering this population of advanced cancer patients, participant burden was a key consideration in our study that should also be taken into account in future investigations.

Our study provides an initial insight into changes in body composition experienced by patients with MPM; however, further research is needed to understand the factors that contribute to the maintenance or decline of physical activity and dietary intake over time. A strength of our study is the use of DXA for body composition analysis, which enabled us to complete a reliable evaluation of appendicular skeletal muscle mass and whole-body and regional fat mass [20]. These data cannot be obtained through computed tomography evaluation of body composition, which employs a single cross-section analysis, and existing 
prediction equations used to convert cross-sectional data to appendicular skeletal muscle mass may be inaccurate [35]. In addition, we report device-derived sedentary behaviour and physical activity using an accelerometer, which has greater accuracy when compared with self-report questionnaires [36].

\section{CONCLUSION}

For the first time, we report on body composition changes over time in patients with MPM. Our results indicate that multiple patterns of change in body composition exist in this patient population. Muscle loss was associated with poorer survival and decreased activity levels. Interventions that improve physical activity or muscle mass could benefit patients with MPM.

\section{REFERENCES}

1. Robinson BWS, Musk AW, Lake RA. Malignant mesothelioma. Lancet. 2005;366:397-408.

2. Finn RS, Brims FJ, Gandhi A, Olsen N, Musk AW, Maskell NA, et al. Postmortem findings of malignant pleural mesothelioma: a two-center study of 318 patients. Chest. 2012;142:1267-73. https://doi.org/10.1378/chest.11-3204.

3. Fearon K, Arends J, Baracos V. Understanding the mechanisms and treatment options in cancer cachexia. Nat Rev Clin Oncol. 2013;10:90-99. https://doi.org/ 10.1038/nrclinonc.2012.209.

4. Fearon $\mathrm{K}$, Arends J, Baracos V. Understanding the mechanisms and treatment options in cancer cachexia. Nat RevBye A, Sjoblom B, Wentzel-Larsen T, Gronberg BH, Baracos VE, Hjermstad MJ, et al. Muscle mass and association to quality of life in non-small cell lung cancer patients. J Cachexia Sarcopenia Muscle. 2017;8:759-67. 10.1002/ jcsm.12206Clin Oncol. 2013;10:90-99. https://doi.org/10.1038/nrclinonc.2012.209.

5. Jeffery E, Lee YCG, Newton RU, Lyons-Wall P, McVeigh J, Nowak AK, et al. Body composition and nutritional status in malignant pleural mesothelioma: implications for activity levels and quality of life. Eur J Clin Nutr. 2019;73:1412-21.

6. Shachar SS, Williams GR, Muss HB, Nishijima TF. Prognostic value of sarcopenia in adults with solid tumours: a meta-analysis and systematic review. Eur J Cancer. 2016;57:58-67. https://doi.org/10.1016/j.ejca.2015.12.030.

7. Baracos VE, Arribas L. Sarcopenic obesity: hidden muscle wasting and its impact for survival and complications of cancer therapy. Ann Oncol.2018;29(Suppl_2): ii1-ii9. https://doi.org/10.1093/annonc/mdx810.

8. Brook MS, Wilkinson DJ, Smith K, Atherton PJ. The metabolic and temporal basis of muscle hypertrophy in response to resistance exercise. Eur J Sport Sci. 2016;16:633-44. https://doi.org/10.1080/17461391.2015.1073362.

9. Steffl M, Bohannon RW, Sontakova L, Tufano JJ, Shiells K, Holmerova I. Relationship between sarcopenia and physical activity in older people: a systematic review and meta-analysis. Clin Inter Aging. 2017;12:835-45. https://doi.org/10.2147/cia.S132940.

10. Houston DK, Nicklas BJ, Ding J, Harris TB, Tylavsky FA, Newman AB, et al. Dietary protein intake is associated with lean mass change in older, community-dwelling adults: the Health, Aging, and Body Composition (Health ABC) Study. Am J Clin Nutr. 2008;87:150-5. https://doi.org/10.1093/ajcn/87.1.150.

11. Jequier E, Tappy L. Regulation of body weight in humans. Physiol Rev. 1999;79:451-80. https://doi.org/10.1152/physrev.1999.79.2.451.

12. Oken MM, Creech RH, Tormey DC, Horton J, Davis TE, McFadden ET, et al. Toxicity and response criteria of the Eastern Cooperative Oncology Group. Am J Clin Oncol. 1982:5:649-55.

13. World Health Organisation. Waist circumference and waist-hip ratio: reports of a WHO expert consultation, 8-11 December 2008. Geneva: World Health Organisation. 2011.

14. Nowitz $M$, Monahan P. Short term in vivo precision of whole body composition measurements on the Horizon A densitometer. J Med Imaging Radiat Oncol. 2018;62:179-82. https://doi.org/10.1111/1754-9485.12646.

15. Centres for Disease Control and Prevention. National Health and Nutrition Examination Survey (NHANES) body composition procedures manual. 2013.

16. Hart NH, Nimphius S, Spiteri T, Cochrane JL, Newton RU. Segmental musculoskeletal examinations using dual-energy X-ray absorptiometry (DXA): positioning and analysis considerations. J Sports Sci Med. 2015;14:620-6.

17. Mourtzakis M, Prado CM, Lieffers JR, Reiman T, McCargar LJ, Baracos VE. A practical and precise approach to quantification of body composition in cancer patients using computed tomography images acquired during routine care. Appl Physiol Nutr Metab. 2008;33:997-1006. https://doi.org/10.1139/h08-075.

18. Kirk B, Bani Hassan E, Brennan-Olsen S, Vogrin S, Bird S, Zanker J, et al. Body composition reference ranges in community-dwelling adults using dual-energy X-ray absorptiometry: the Australian Body Composition (ABC) Study. J Cachexia Sarcopenia Muscle. 2021;12:880-90. https://doi.org/10.1002/jcsm.12712.
19. Cruz-Jentoft AJ, Bahat G, Bauer J, Boirie Y, Bruyere O, Cederholm T, et al. Sarcopenia: revised European consensus on definition and diagnosis. Age Ageing. 2019;48:16-31. https://doi.org/10.1093/ageing/afy169.

20. Prado CM, Heymsfield SB. Lean tissue imaging: a new era for nutritional assessment and intervention. JPEN J Parenter Enter Nutr. 2014;38:940-53. https:// doi.org/10.1177/0148607114550189.

21. Santos-Lozano A, Marín PJ, Torres-Luque G, Ruiz JR, Lucía A, Garatachea N. Technical variability of the GT3X accelerometer. Med Eng Phys. 2012;34:787-90. https://doi.org/10.1016/j.medengphy.2012.02.005.

22. Freedson PS, Melanson E, Sirard J. Calibration of the Computer Science and Applications, Inc. accelerometer. Med Sci Sports Exerc. 1998;30:777-81.

23. Matthews CE, Chen KY, Freedson PS, Buchowski MS, Beech BM, Pate RR, et al. Amount of time spent in sedentary behaviors in the United States, 2003-2004. Am J Epidemiol. 2008;167:875-81. https://doi.org/10.1093/aje/kwm390.

24. Gariballa SE, Forster SJ. Dietary intake of older patients in hospital and at home: the validity of patient kept food diaries. J Nutr Health Aging. 2008;12:102-6. https://doi.org/10.1007/bf02982561.

25. Conway JM, Ingwersen LA, Moshfegh AJ. Accuracy of dietary recall using the USDA five-step multiple-pass method in men: an observational validation study. J Am Diet Assoc. 2004;104:595-603. https://doi.org/10.1016/j.jada.2004.01.007.

26. Prado CM, Sawyer MB, Ghosh S, Lieffers JR, Esfandiari N, Antoun S, et al. Central tenet of cancer cachexia therapy: do patients with advanced cancer have exploitable anabolic potential? Am J Clin Nutr. 2013;98:1012-9. https://doi.org/ 10.3945/ajcn.113.060228.

27. Argiles JM, Busquets S, Stemmler B, Lopez-Soriano FJ. Cancer cachexia: understanding the molecular basis. Nat Rev Cancer. 2014;14:754-62. https://doi.org/ $10.1038 / \mathrm{nrc3829}$.

28. Vogelzang NJ, Rusthoven JJ, Symanowski J, Denham C, Kaukel E, Ruffie $P$, et al. Phase III study of pemetrexed in combination with cisplatin versus cisplatin alone in patients with malignant pleural mesothelioma. J Clin Oncol. 2003;21:2636-44. https://doi.org/10.1200/jco.2003.11.136.

29. Peddle-McIntyre C, Jeffery E, Lee YCG, Nowak A, McVeigh J, Nguyen B, et al. Resistance exercise training improves physical functioning and sarcopenia status in patients with malignant pleural disease. In preparation.

30. Arends J, Bachmann P, Baracos V, Barthelemy N, Bertz H, Bozzetti F, et al. ESPEN guidelines on nutrition in cancer patients. Clin Nutr. 2017;36:11-48. https://doi. org/10.1016/j.clnu.2016.07.015.

31. Tobberup R, Rasmussen HH, Holst M, Jensen NA, Falkmer UG, Bogsted M, et al. Exploring the dietary protein intake and skeletal muscle during first-line antineoplastic treatment in patients with non-small cell lung cancer. Clin Nutr Espen. 2019;34:94-100. https://doi.org/10.1016/j.clnesp.2019.08.006.

32. Ulmann G, Jouinot A, Tlemsani C, Curis E, Kousignian I, Neveux N, et al. Lean body mass and endocrine status but not age are determinants of resting energy expenditure in patients with non-small cell lung cancer. Ann Nutr Metab. 2019;75:223-30. https://doi.org/10.1159/000504874.

33. Cao DX, Wu GH, Zhang B, Quan YJ, Wei J, Jin $\mathrm{H}$, et al. Resting energy expenditure and body composition in patients with newly detected cancer. Clin Nutr. 2010;29:72-7. https://doi.org/10.1016/j.clnu.2009.07.001.

34. Engelen MP, van der Meij BS, Deutz NE. Protein anabolic resistance in cancer: does it really exist? Curr Opin Clin Nutr Metab Care. 2016;19:39-47. https://doi. org/10.1097/mco.0000000000000236.

35. Kilgour RD, Cardiff K, Rosenthall L, Lucar E, Trutschnigg B, Vigano A. Use of prediction equations to determine the accuracy of whole-body fat and fat-free mass and appendicular skeletal muscle mass measurements from a single abdominal image using computed tomography in advanced cancer patients. Appl Physiol Nutr Metab. 2016;41:70-5. https://doi.org/10.1139/apnm-2015-0068.

36. Cleland C, Ferguson S, Ellis G, Hunter RF. Validity of the International Physical Activity Questionnaire (IPAQ) for assessing moderate-to-vigorous physical activity and sedentary behaviour of older adults in the United Kingdom. BMC Med Res Methodol. 2018;18:176. https://doi.org/10.1186/s12874-018-0642-3.

\section{AUTHOR CONTRIBUTIONS}

EJ, YCGL, RUN, PL-W and CJP-M contributed to the conception of the design of the study. EJ and DBF collected the data. EJ, JM and LS contributed to data analysis. All authors contributed to the article and have approved the submission of the article for publication.

\section{FUNDING}

EJ was supported by an Australian Lung Foundation/Edith Cowan University PhD Scholarship and Australian Government Research Training Program Scholarship. CJP$M$ was supported by a Cancer Council Western Australia Postdoctoral Fellowship. Open Access funding enabled and organized by CAUL and its Member Institutions. 


\section{COMPETING INTERESTS}

The authors declare no competing interests.

\section{ADDITIONAL INFORMATION}

Correspondence and requests for materials should be addressed to Emily Jeffery.

Reprints and permission information is available at http://www.nature.com/ reprints

Publisher's note Springer Nature remains neutral with regard to jurisdictional claims in published maps and institutional affiliations.
Open Access This article is licensed under a Creative Commons Attribution 4.0 International License, which permits use, sharing, adaptation, distribution and reproduction in any medium or format, as long as you give appropriate credit to the original author(s) and the source, provide a link to the Creative Commons license, and indicate if changes were made. The images or other third party material in this article are included in the article's Creative Commons license, unless indicated otherwise in a credit line to the material. If material is not included in the article's Creative Commons license and your intended use is not permitted by statutory regulation or exceeds the permitted use, you will need to obtain permission directly from the copyright holder. To view a copy of this license, visit http://creativecommons.org/licenses/by/4.0/.

(c) The Author(s) 2022 